\title{
CAMPTOCORMIA: A RARE AXIAL MYOPATHY DISEASE
}

doi: $10.1590 / \mathbf{S 1 8 0 7 - 5 9 3 2 2 0 0 8 0 0 0 3 0 0 0 2 4}$

\section{Samuel Katsuyuki Shinjo, Silvia Carolina Ramos Torres, Ari Stiel Radu}

\section{INTRODUCTION}

Camptocormia [from the Greek words: kamptos (to bend) and kormos (trunk)] is characterized by an abnormal posture of the trunk; the condition involves forced thoraco-lumbar flexion that increases during walking and disappears in the recumbent position.

Camptocormia was first described by Brodie in $1818,{ }^{2,3}$ who suggested that lumbar pain and abnormal curvature of the spine might be caused both by destruction of the vertebrae and by hysterical reactions. In 1915, the term "camptocormia" was proposed for this abnormal posture. ${ }^{4}$

Although early cases were assumed to be psychogenic in origin, several reports of organic causes of camptocormia have been described in lesions affecting the lenticular nucleus, ${ }^{5}$ Parkinson's disease,${ }^{6,7}$ dystonia, ${ }^{8}$ and neuromuscular disorders, ${ }^{9,10}$ as well as paraneoplastic syndrome, ${ }^{11}$ inclusion body myositis, ${ }^{12}$ drug-induced camptocormia, ${ }^{13}$ and Grave's disease. ${ }^{14}$

This present case provides clear evidence for camptocormia of organic rather than psychogenic origin.

\section{CASE DESCRIPTION}

A 78-year-old Japanese female patient presented with a complaint of lower back pain. She reported that from the age of 66, she had found it progressively more difficult to maintain an erect orthostatic posture. She reported no family history of similar disorders.

Gait analysis showed trunk flexion and drooping arms (Figure 1A). In contrast, when she was allowed to hold onto a table, she could maintain an erect posture; in addition,

Serviço de Reumatologia, Faculdade de Medicina da Universidade de São Paulo - São Paulo. Brazil.

samuel.shinjo@gmail.com
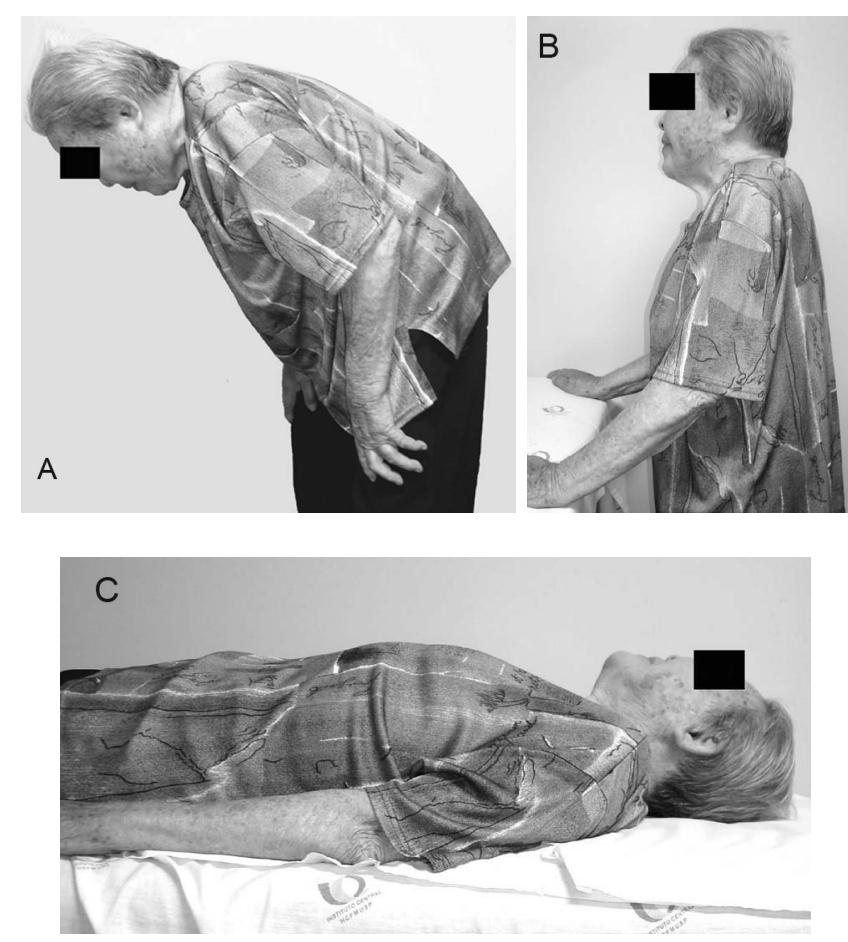

Figure 1 - Patient with camptocormia (A) whose posture markedly improved when when she was allowed to hold onto a table, (B) or when she lay in a supine position $(\mathrm{C})$

the thoraco-lombar spine flexion disappeared when she lay down (Figures 1B and 1C). Lower limb strength was normal. Physical examination of the spine was normal, without scoliosis, cifosis, or lordosis. There were no signs or symptoms of any primary neurologic pathology.

A computerized tomography (CT) scan of the lower lumbarand sacral spine showed usualdegenerative changes in intervertebral disks, facets, and vertebrae as well asparaspinal muscle atrophy.

An electrophysiological study revealed severe chronic symmetric myopathy affecting the lumbosacral paravertebral 
muscle, and no abnormalities of the lower extremities.

A localized muscle biopsy revealed muscle tissue atrophy without any inflammation.

Laboratory analysis gave results within normal ranges.

The patient received conservative treatment with orthesis and physiotherapy support, and the level of back pain was stabilized.

\section{DISCUSSION}

Camptocormia is a syndrome confined to the extensor muscles of the spine. There are usually no other motor or sensory symptoms. Lumbar radicular nerves are unaffected. However, some patients may suffer mild discomfort in the lower back. Moreover, some authors suggest that symptoms may not occur unless there is also hip flexion contracture. ${ }^{16}$

Radiological analysis of the thoraco-lumbar spine by MRI or CT can reveal paraspinal muscle atrophy heterogeneous in appearance, with progressive replacement of paraspinal muscles with fat ${ }^{17}$ Electromyography findings are not uniform. Both myogenic and neurogenic features of the disease have been reported. ${ }^{16}$

Unfortunately, effective treatment is unavailable. In very few cases, patients respond to electrotherapy or to corticosteroid medication, but most cases fail to respond to any treatment. ${ }^{18}$ Deep brain stimulation of the medial globus pallidus interna has been claimed to produce a gradual but partial improvement, which supports the notion of segmental dystonia. ${ }^{18}$ Some pharmacologic treatments attempted so far include anticholinergics, amantadine, dopamine agonists, muscle relaxants, and tetrabenazine, but these have not led to improvement in posture. ${ }^{1}$

In the present case, the patient received conservative treatment, with orthesis and physiotherapy support, and the back pain stopped worsening.

\section{REFERENCES}

1. Azher SN, Jankovic J. Camptocormia. Pathogenesis, classification, and response to therapy. Neurology. 2005;65:355-9.

2. Brodie BC. Pathological and surgical observations on the diseases of the joints. London: Longman; 1818. p. 276.

3. Brodie BC. Lectures illustrative of certain local nervous affections. London: Longman, 1837. Cited according to Hawkins C. The Works of Sir Benjamin Collins Brodie, Vol 3. London: Longman; 1865. p.164.

4. Souques A, Rosanoff-Saloff. La camptocormie; incurvation du tronc, consecutive aux traumatismes du dos et des lombes; considerations morphologiques. Rev Neurol. 1914;15:937-9.

5. Nieves AV, Miyasaki JM, Lang AE. Acute onset dystonic camptocormia caused by lenticular lesions. Mov Disord. 2001;16:177-80.

6. Djaldetti R, Mosberg-Galili R, Sroka H, Merims D, Melamed E. Camptocormia (bent spine) in patients with Parkinson's diseasecharacterization and possible pathogenesis of an unusual phenomenon. Mov Disord. 1999;14:443-7.

7. Schabitz WR, Glatz K, Schuhan C, Sommer C, Berger C, Schwaninger $\mathrm{M}$, et al. Severe forward flexion of the trunk in Parkinson's disease: focal myopathy of the paraspinal muscles mimicking camptocormia. Mov Disord. 2003;18:408-14.

8. Reichel G, Kirchhofer U, Stenner A. Camptocormia - segmental dystonia. Proposal of a new definition for an old disease. Nervenarzt. 2000;72:281-5.

9. Laroche M, Delisle MB, Aziza R, Lagarrigue J, Mazieres B. Is camptocormia a primary muscular disease? Spine. 1995;20:1011-6.
10. Wunderlich S, Csoti I, Reiners K, Günthner-Lengsfeld T, Schneider C, Becker G, et al. Camptocormia in Parkinson's disease mimicked by focal myositis of the paraspinal muscles. Mov Disord. 2002;17:598-600.

11. Zwecker M, Iancu I, Zeilig G, Ohry A. Camptocormia: a case of possible paraneoplastic aetiology. Clin Rehabil. 1998;12:157-60.

12. Hund E, Heckl R, Goebel H, Meinck HM. Inclusion body myositis presenting with isolated erector spinae paresis. Neurology. 1995;45:993-4.

13. Kiuru S, Iivanainen M. Camptocormia, a new side effect of sodium valproate. Epilepsy Res. 1987;1:254-7.

14. Loh LM, Hum AY, Teoh HL, Lim EC. Graves' disease associated with spasmodic truncal flexion. Parkinsonism Relat Disord. 2005;11:117-9.

15. Karbowski K. The old and new camptocormia. Spine. 1999;24:1494-8.

16. Haig AJ, Tong HC, Kendall R. The bent spine syndrome: myopathy biomechanics symptoms. Spine J. 2006;6:190-4.

17. Umapathi T, Chaudhry V, Comblath D, Drachman D, Griffin J, Kuncl R. Head drop and camptocormia. J Neurol Neurosurg Psychiatry. 2002;73:1-7.

18. Nandi D, Parkin S, Scott R, Winter JL, Joint C, Gregory R. Camptocormia treated with bilateral pallidal stimulation. J Neurosurg. 2002;97:461-6. 